\title{
Frailty and Biological Age
}

\author{
Lixin $\mathrm{Ji}^{1}$, S. Michal Jazwinski ${ }^{2}$, Sangkyu Kim ${ }^{2}$ \\ ${ }^{1}$ Tulane University School of Medicine, New Orleans, LA, USA \\ ${ }^{2}$ Tulane Center for Aging \& Department of Medicine, Tulane University Health Sciences Center, New Orleans, LA, USA
}

\section{Corresponding Author:}

Sangkyu Kim, PhD

Tulane Center for Aging \& Department

of Medicine, Tulane University Health

Sciences Center, New Orleans,

Louisiana, USA

E-mail: skim5@tulane.edu

ORCID:

https://orcid.org/0000-0001-6761-2579

Received: July 26, 2021

Accepted: August 10, 2021
A reliable model of biological age is instrumental in the field of geriatrics and gerontology. This model should account for the heterogeneity and plasticity of aging and also accurately predict aging-related adverse outcomes. Epigenetic age models are based on DNA methylation levels at selected genomic sites and can be significant predictors of mortality and healthy/unhealthy aging. However, the biological function of DNA methylation at selected sites is yet to be determined. Frailty is a syndrome resulting from decreased physiological reserves and resilience. The frailty index is a probability-based extension of the concept of frailty. Defined as the proportion of health deficits, the frailty index quantifies the progression of unhealthy aging. The frailty index is currently the best predictor of mortality. It is associated with various biological factors and provides insight into the biological processes of aging. Investigation of the multi-omics factors associated with the frailty index will provide further insight.

Key Words: Aging, Healthy aging, Frailty, Gene expression, DNA Methylation

\section{INTRODUCTION}

As we age, our ability to withstand damage and stress declines, and the incidence of disability, disease, and mortality increases. ${ }^{1)}$ Time underlies all these biological processes and events. As it is universal and readily available, calendar age is a popular estimate of the rate of biological aging in daily life. Indeed, as living matter constantly and dynamically changes with time, numerous age-related changes occur throughout all organizational levels, from molecular to organismal to populational levels. With increasing age, however, age-related changes occur at varying rates. ${ }^{2)}$ For example, older individuals of a certain chronological age differ in their physical and cognitive functioning. Thus, biological aging is heterogeneous and often disproportionate with the amount of time passed.

In addition to heterogeneity, plasticity is another property of biological aging that chronological age cannot accommodate. Tales abound of a Fountain of Youth or a Chinese emperor who sought an elixir of life that would enable him to live forever. Experimental evidence suggests that these mythical or legendary tales may not be entirely baseless. The pace of aging may be delayable or even re- versible. The evidence is mostly based on genetic or interventional studies using model organisms such as yeast, fruit flies, nematodes, mice, and even monkeys. ${ }^{3-5)}$ When mutated, many genes can modify the lifespans of non-mammalian model organisms. Some of these genes include SIR2 in brewer's yeast, ${ }^{6}$ ) daf-2 in nematodes, ${ }^{7)}$ and methuselah in fruit flies. ${ }^{8)}$ Gene therapy using genes involved in aging or age-related disease extended both lifespan and health span in mice. ${ }^{9)}$ Pharmaceutical and nutritional interventions, such as the administration of the immunosuppressive drug rapamycin and calorie/dietary restriction, also significantly extended the lifespan and improved the fitness or health of model organisms. ${ }^{3)}$

Biological age is equivalent to physiological or functional age, whereas chronological age is physical or mathematical. As aging progresses, mortality increases exponentially not because time passes at an exponential speed but because aging-related detrimental events occur exponentially. The biology of aging concerns the biological processes of aging, which occur separately from the invariant passage of time. Thus, a reliable measure of biological age will better suit our pursuit of precision gerontology or personalized geriatrics. While the hallmarks of aging have been described, ${ }^{10)}$ the 
mechanisms underlying biological aging remain largely unknown. One approach to the biology of aging is to model biological age and investigate its associated factors.

\section{MODELS OF BIOLOGICAL AGE}

Different types of cells age differently. ${ }^{11,12)}$ Heterogeneity in tissue aging contributes to the heterogeneity of aging in different organs and body systems. Thus, no single biological marker of aging can faithfully represent organismal aging. Biomarkers of aging have long been sought from age-related changes; however, the relevance of all age-related changes to the biology of aging remains unknown. As some age-related changes may not be relevant to the biology of aging while others constitute the true mechanisms of aging, multiple biomarkers of aging are used to estimate or models the true biological age. Epigenetic age-based models of biological age were first proposed a decade ago, and all such models are based on DNA methylation data. ${ }^{13)}$ Another type of biological age models that has been gaining momentum is based on frailty. ${ }^{14)}$ These frailty-based models use health deficits or health-related changes found in aging individuals. We will first discuss models based on epigenetic age before reviewing frailty-based models of biological age.

\section{Epigenetic Age Models}

Genomic DNA methylation sites that we are currently focusing on are conveniently called $\mathrm{CpG}$ sites as the methylation of cytosine occurs predominantly in $\mathrm{CpG}$ dinucleotides ("p" represents the phosphodiester bond linking the nucleotides). A recent study compiled 11 epigenetic age models, ${ }^{13)}$ one of which was based on 71 CpG sites in the leukocyte genome. ${ }^{15)}$ These CpGs were selected using a regularized regression method, which showed that their DNA methylation levels were highly correlated with chronological age. The predicted epigenetic age was obtained using actual methylation data in the regression model. Individuals with epigenetic ages greater and less than their calendar ages are categorized as fast aging and slow aging, respectively. Similarly, DNA methylation age and related measures were calculated based on $353 \mathrm{CpGs}$ whose methylation levels were highly correlated with chronological age in multiple tissues. ${ }^{16)}$ A change in epigenetic age depends on a change in the methylation levels of selected CpGs. As shown in Fig. 1, a decrease in the overall DNA methylation level of $353 \mathrm{CpGs}$ resulted in decreased epigenetic age. Since the genomic DNA methylation level tends to decrease with increasing chronological age, ${ }^{17-19)}$ the epigenetic age is likely to decrease with increasing chronological age among the oldest of older individuals. ${ }^{20)}$

Recent epigenetic age models combine health-related data with DNA methylation data using complex multi-step mathematical or statistical approaches. "DNAm PhenoAge" is based on 513 CpGs associated with the Gompertz model-based phenotypic age. ${ }^{21)}$ This phenotypic age incorporates laboratory blood test findings and chronological age. Other models use $\mathrm{CpGs}$ as proxy markers for age-related changes or biological events. For example, age-correlated CpGs have been combined with biological data to build a mitotic-like clock model to predict cancer risk. ${ }^{22}$ " "GrimAge" uses $\mathrm{CpGs}$ that are selected by regressing plasma proteins and smoking on 485,000 CpG sites and chronological age. ${ }^{23)}$

Epigenetic-based models of age are associated with mortality. ${ }^{24-26)}$ Slow epigenetic aging is associated with healthy aging-related factors such as healthy diet, physical activity, lifestyle, and low morbidity. ${ }^{23,27,28)}$ This is potentially interesting in that healthy aging is determined largely by non-genetic factors, including various environmental factors and lifestyle, and DNA methylation is an epigenetic interface between genes and non-genetic determinants. Nevertheless, the biological function of the DNA methylation level in CpGs used in the models remains unknown.

The human genome contains an estimated 28 million CpGs. ${ }^{29)}$ A cross-sectional compilation found that approximately $11 \%$ of 485,577 CpGs examined were significantly correlated with chronological age and approximately $30 \%$ of them also significantly changed longitudinally. ${ }^{30}$ If we assume random sampling of CpGs examined, the DNA methylation of approximately 3 million

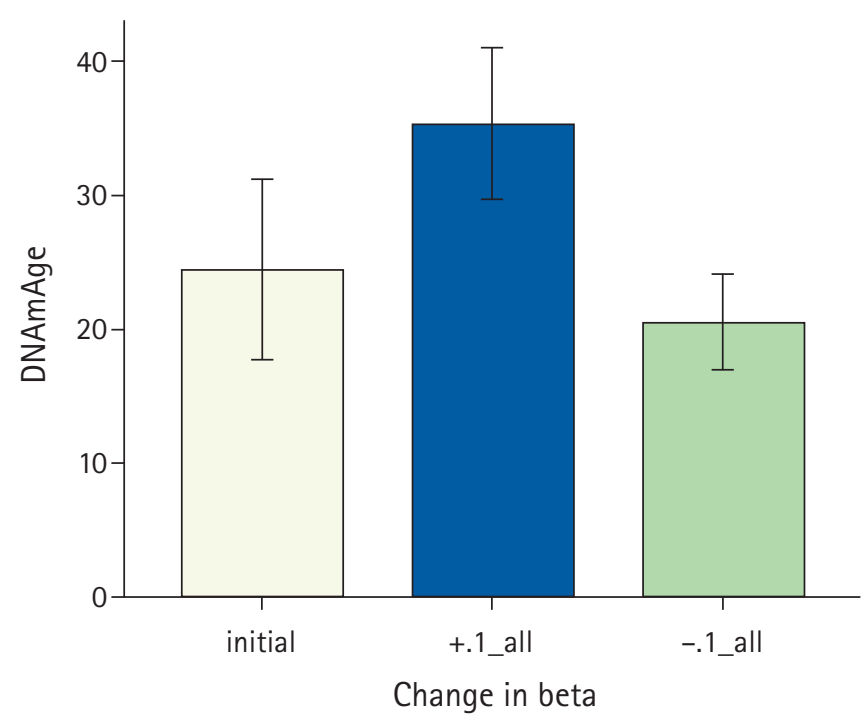

Fig. 1. A bar plot of mean DNA methylation ages and standard errors. This plot was generated using the data from eight autistic subjects available in the MethylationDataExample55 and SampleAnnotationExample55 datasets provided. ${ }^{16)}$ DNA methylation age was calculated in $\mathrm{R}$ using the tutorial provided. The y-axis shows mean DNAmAge \pm standard error corresponding to three groups on the $\mathrm{x}$-axis: the autistic subjects ("initial"), after adding 0.1 to the original beta values of 353 CpGs ("+.1_all”), or subtracting 0.1 from the original beta values of 353 CpGs (“-1_all”). 
CpGs in the genome is correlated with chronological age, and 0.9 million of them will change longitudinally. With that many age-associated $\mathrm{CpGs}$, one can select any subset of $\mathrm{CpGs}$ to predict chronological age or any age-associated changes. One can even select a subset of $\mathrm{CpGs}$ in the genome to predict any randomly generated numeric variable (Table 1 ). Note that in Table 1 , the variable importance scores generated using a random forest method for the randomly generated numeric variable are comparable to those for the frailty index. Similar results were obtained using the regularized regression method. The abundance of $\mathrm{CpGs}$ associated with chronological age may also explain why subsets of selected CpGs used in epigenetic age models overlap sparsely (Fig. 2). Thus, the biological roles of these CpGs, if any, remain unknown.

\section{Biological Functions of CpG Sites Associated with Age}

DNA methylation affects important biological phenomena such as gene expression, DNA imprinting, X-chromosome inactivation, and genome stability. ${ }^{31}$ Of these, differential gene expression is most relevant to the biology of aging. Changes in the other phenomena tend to have drastic, often pathological, effects, although their contributions to the biology of aging cannot be completely ruled out.

The transcriptional regulation of gene expression is primarily mediated by DNA sequence-based genetic factors. DNA methylation also affects gene transcription via epigenetic mechanisms. Following the erasure of DNA methylation patterns of the previous generation, genomic DNA of fertilized eggs is methylated de novo in a tissue-specific manner during implantation and differentia- tion-the erasure seems incomplete, which explains in part why DNA methylation is heritable. DNA methylation may serve as a safety lock to ensure the long-term repression of certain genes. ${ }^{34)}$ For genes whose ${ }^{32,33)}$ expression is responsive to environmental

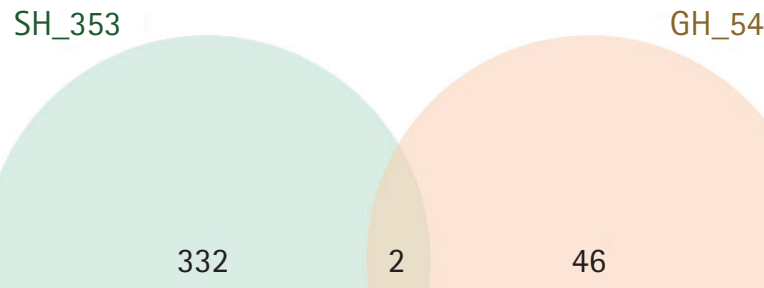

11 6 983

Fig. 2. A Venn diagram of three sets of genes. SH_353 represents genes annotated for $353 \mathrm{CpGs}$ used in the DNA methylation age model. ${ }^{16)} \mathrm{GH}$ 54 represents genes annotated for $71 \mathrm{CpGs}$ used to determine the apparent methylomic aging rate. ${ }^{15)}$ MS.VG_1000 represents top 1,000 genes whose transcript levels are important in predictive modeling of chronological age. ${ }^{37)}$

Table 1. Top 10 DNA methylation sites associated with the prediction of age, frailty index, or a sequence of randomly generated numbers

\begin{tabular}{|c|c|c|c|c|c|}
\hline \multicolumn{2}{|c|}{$\operatorname{Age}^{\mathrm{a})}$} & \multicolumn{2}{|c|}{ FI34 ${ }^{\mathrm{b})}$} & \multicolumn{2}{|c|}{ Random number ${ }^{c)}$} \\
\hline CpG site ${ }^{\text {d) }}$ & Importance $^{\mathrm{e})}$ & CpG site $^{\mathrm{d})}$ & Importance $^{\mathrm{e})}$ & CpG site ${ }^{\mathrm{d})}$ & Importance $^{\mathrm{e})}$ \\
\hline $\operatorname{cg} 18404925$ & 30.4 & $\operatorname{cg} 10458216$ & 12.9 & $\operatorname{cg} 02271560$ & 12.0 \\
\hline cg21070864 & 27.0 & $\operatorname{cg} 26188685$ & 4.6 & $\operatorname{cg} 10332616$ & 7.9 \\
\hline cg26811352 & 14.9 & $\operatorname{cg} 01290856$ & 4.4 & $\operatorname{cg} 26565544$ & 4.9 \\
\hline $\operatorname{cg} 16624788$ & 14.5 & $\operatorname{cg} 23765086$ & 4.2 & $\operatorname{cg} 00981070$ & 4.7 \\
\hline cg01290856 & 12.7 & $\operatorname{cg} 11177404$ & 4.1 & $\operatorname{cg} 12645852$ & 4.3 \\
\hline $\operatorname{cg} 13480818$ & 10.9 & $\operatorname{cg} 02716646$ & 3.9 & $\operatorname{cg} 04365609$ & 3.7 \\
\hline $\operatorname{cg} 03767951$ & 9.7 & $\operatorname{cg} 10043954$ & 3.9 & $\operatorname{cg} 05461182$ & 3.7 \\
\hline $\operatorname{cg} 01874084$ & 9.5 & cg21561157 & 3.8 & $\operatorname{cg} 05935052$ & 3.6 \\
\hline cg07935657 & 7.3 & $\operatorname{cg} 10223982$ & 3.2 & $\operatorname{cg} 03475190$ & 3.5 \\
\hline $\operatorname{cg} 13567404$ & 7.2 & $\operatorname{cg} 05304366$ & 3.2 & $\operatorname{cg} 10697491$ & 3.4 \\
\hline
\end{tabular}

a) The calendar ages (60-103 years) of 211 participants in the Louisiana Healthy Aging Study ${ }^{72)}$ were regressed on DNA methylation values (beta) of 10,000 randomly selected $\mathrm{CpG}$ sites using a random forest regression method in $\mathrm{R}$.

b) The same random forest as in ${ }^{\text {a) }}$, with a frailty index, FI34, instead of calendar age.

${ }^{c)}$ The same random forest as in ${ }^{\text {a) }}$, with a variable of randomly generated numbers instead of calendar age.

${ }^{\mathrm{d})}$ Probes used to assess DNA methylation levels of the CpG sites using the Infinium HumanMethylation450K BeadChip Kit (Illumina Inc., San Diego, CA, USA).

${ }^{\text {e) }}$ Scaled permutation-based variable importance scores calculated using the random forest. 
cues, DNA methylation is also a way of non-genetically attuning gene expression to changing milieus. In general, hypermethylation is associated with no or lower gene transcription and hypomethylation with higher transcription, although deviant cases exist. ${ }^{35,36)}$

In view of DNA methylation as a modulator of gene transcription, it is interesting to determine whether age-associated DNA methylation of CpGs used in some of the epigenetic age models also show age-associated differential gene transcription. If DNA methylation of $\mathrm{CpGs}$ is strongly associated with chronological age, transcription of the genes annotated for CpGs may be strongly associated with chronological age. A recent comprehensive compilation of multi-tissue transcriptomic data ${ }^{37)}$ excluded genes with low expression to analyze a total of 13,388 genes to determine differentially expressed genes (DEGs) that were important in predictive modeling of chronological age. Among the 54 genes annotated for the $72 \mathrm{CpGs}$ described above, only six ranked in the top 1,000 DEGs associated with chronological age. Similarly, among the 353 genes annotated for the $353 \mathrm{CpGs}$, only 11 ranked in the top 1,000 DEGs (Fig. 2). These results indicated that the transcription of most genes annotated in the model CpGs was not highly associated with chronological age or was not important in the predictive modeling of chronological age. In other words, these findings are at odds with the expected biological role of DNA methylation. Thus, no convincing evidence exists to support the mainstream role of DNA methylation in $\mathrm{CpG}$ sites used in some of the epigenetic age models.

\section{FRAILTY}

Common among older adults, frailty is often used interchangeably with disability, comorbidity, or even old age. One practicable definition of frailty is age greater than 65 years and dependence on others to perform activities of daily living. ${ }^{38)}$ Disability is defined as the inability to perform activities of daily living, while frailty along with other morbidities contributes to disability. ${ }^{39)}$ Frailty may develop from diminished physiological reserve before disability, comorbidity, or other adverse outcomes. ${ }^{40)}$ Frailty is a multidimensional phenotype that manifests through various signs, symptoms, or other health-related events. ${ }^{41)}$ To operationalize frailty as a clinical syndrome, the Fried frailty uses five phenotypic criteria: (1) weight loss (> $10 \mathrm{lb}$ in the previous year); (2) self-reported exhaustion; (3) muscle weakness (the lowest quintile of hand grip strength); (4) slow gait speed (the lowest quintile in walking time per 15 feet); and (5) low physical activity (the lowest quintile in kilocalories expended per week). Individuals meeting three or more of these criteria are considered frail. Related to the Fried frailty, Edmonton frailty uses nine criteria: cognition, general health status, functional independence, social support, medication use, nutrition, mood, continence, and functional performance. ${ }^{42)}$ The maximum score of Edmonton frailty is 17.

Geriatric status classification in clinical settings requires a streamlined operationalization of frailty. Based on activities of daily living, a clinical frailty scale has been proposed to classify older people. ${ }^{43)}$ A recent version of the Clinical Frailty Scale (CFS) has nine categories, from "very fit" to "terminally ill." ${ }^{44)}$ It relies on the level of dependence in daily living activities and clinical judgment. The CFS is simple, inexpensive, and easy to perform but has been criticized as potentially subjective. However, a recent study of the CFS reported a general agreement of participants in diverse medical professions and health occupations in the classification of scenario cases. ${ }^{45)}$ The CFS was a significant predictor of survival of patients with coronavirus disease (COVID-19). ${ }^{46)}$ and the outcome of coronary artery bypass grafting. ${ }^{47)}$

\section{FRAILTY/DEFICIT INDEX}

The criteria for frailty scales described above yield semiquantitative measures. By including more health items and using a continuous scoring method, one can build a fully quantitative model of frailty. The frailty index is defined as the proportion of health deficits among a set of surveyed health items. ${ }^{48,49)}$ The deficit index uses the same calculation method as the frailty index. ${ }^{50)}$ The health items include various symptoms, signs, disabilities, and diseases. ${ }^{48)}$ These data are collected from medical history questionnaires, clinical assessments, laboratory measurements, and other data collection instruments. As the proportion of health deficits that an aging individual carries at the time of survey, raw frailty index scores range from 0 (no deficit in all health items surveyed) to 1 (at least one problem in every health item). Unlike frailty scales, calculation of the frailty index does not require a fixed set of specific health items. When the number of health items is sufficiently large (typically $\geq 20$ ), different frailty indices based on different sets of health items yield comparable properties and performances. However, including health items with predictive power can significantly improve the performance of a frailty index. ${ }^{51)}$

\section{Frailty Index as a Model of Biological Age}

The end of aging is death, and the oldest of older adults (those aged $>90$ years) are most prone to this and other adverse outcomes. The frailty index shows an age-dependent exponential increase with mortality (Fig. 3). Frailty index scores vary among aged peers and may decline individually while their group average increases over time, accounting for the heterogeneity and plasticity of aging. ${ }^{52)}$ In the general population, chronological age is a strong 

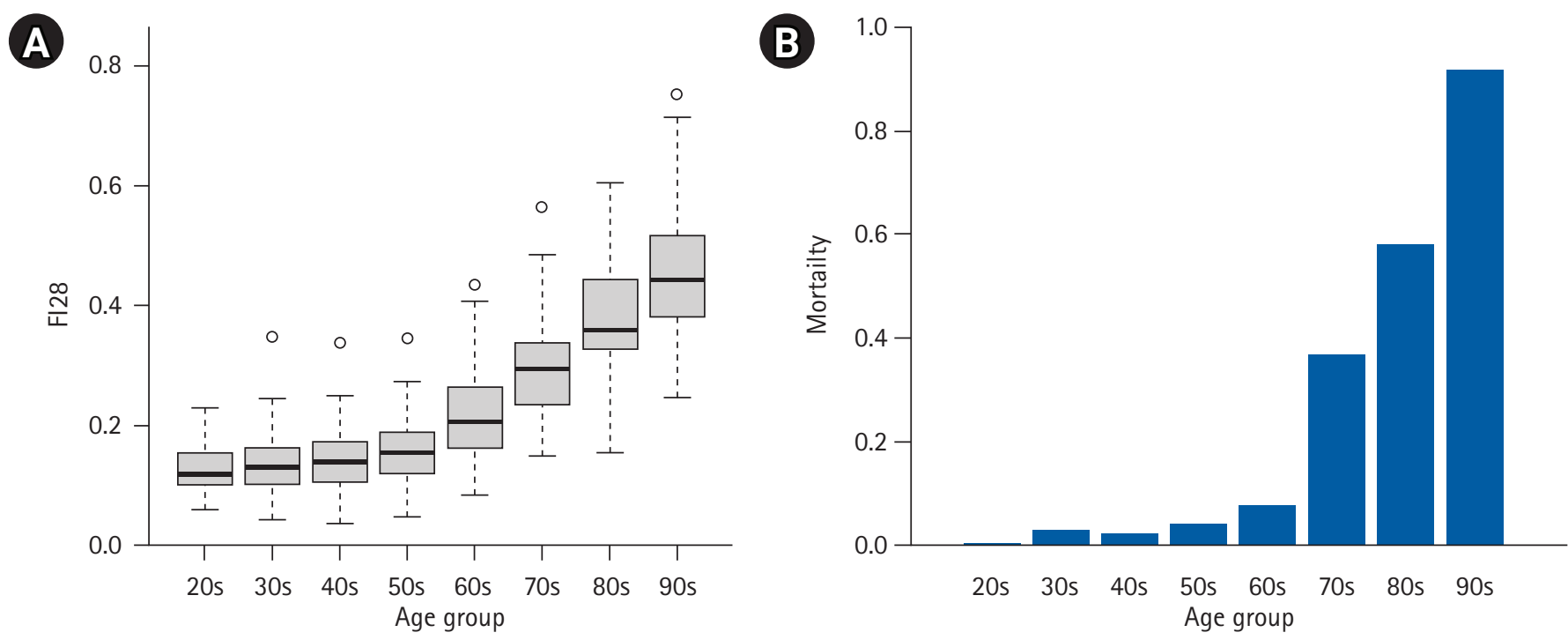

Fig. 3. Exponential increase in the frailty index and mortality. (A) Box plot of FI28 scores of 592 individuals in the Louisiana Healthy Aging Study. ${ }^{51)}$ FI28 is a frailty index based on 28 health items. Each box represents an inter-quartile range with the line in the middle showing the median position. (B) Proportions of the deceased in the same age groups.

predictor of mortality and other time-dependent phenomena. The Klemera-Doubal models of biological age, termed therein Equation 25 (BE) and Equation 34 (BEC), outperformed chronologi$\mathrm{cal}$ age and biological age measures based on multiple linear regression or principal component analysis in predicting mortality. ${ }^{53,54)}$ This finding makes sense in that both health data and chronological age are used in the derivation of $\mathrm{BE}$ and BEC. In particular, BEC explicitly incorporates chronological age as an additional biomarker to improve its performance over $\mathrm{BE}$. After adjusting for age and sex, however, the frailty index outperformed not only DNA methylation age but also both $\mathrm{BE}$ and $\mathrm{BEC}$ in predicting mortality in the oldest of older adults (Fig. 4). Thus, the frailty index is the best predictor of age-related mortality.

\section{Biological Factors Associated with Frailty or Frailty Indexes}

The frailty index quantifies the extent of unhealthy aging. In other words, it is an unhealthy aging index (and subtraction of it from 1 gives a healthy aging index). Therefore, examining biological factors associated with the frailty index can provide insights into the biological mechanisms of unhealthy (or healthy) aging.

The resting metabolic rate (RMR) estimates the amount of energy used for the maintenance of body systems under resting conditions. RMR, which comprises $60 \%-70 \%$ of the total daily energy expenditure, decreases with increasing age in the general population. ${ }^{55)}$ In the oldest of older adults, however, RMR increases as the frailty index increases. ${ }^{56)}$ One interpretation of this is that a higher RMR is required for this population to maintain homeodynamics as their health deteriorates. Some factors also show sex specifici- ties; for instance, the association of frailty index with body composition (fat and fat-free mass) is specific to female nonagenarians, whereas circulating creatine kinase $(\mathrm{CK})$ is specific to male nonagenarians. Detailed analyses of these sex specificities led to the identification of the associations of UCP2 and UCP3 with frailty index in women ${ }^{57)}$ and XRCC6 and LASS1 in men. ${ }^{58)}$ UCP2 and $U C P 3$, which encode uncoupling proteins in the mitochondria, function as metabolite transporters important in energy metabolism. In contrast, XRRC6 and LASS1, which encode the protein $\mathrm{Ku} 70$ and ceramide synthase, respectively, are involved in programmed cell death. Muscle damage caused by strenuous physical activity or exercise is a major factor leading to elevated blood CK levels. ${ }^{59)}$ Thus, one interpretation of the male-specific findings is that an elevated CK level reflects an increased number of damaged muscle cells undergoing programmed death. ${ }^{58}$

The genetic basis of longevity is not as substantial as previously estimated, indicating that non-genetic and environmental factors are much more influential in aging. ${ }^{60)}$ Frailty is associated with various transcriptomic, proteomic, and metabolomic factors. ${ }^{61-68)}$ Overall, the number and species of identified omics factors vary across studies and no omics factors have been convincingly replicated in multiple independent studies. Nevertheless, several studies merit further investigation. $\mathrm{CK}$ is a major protein associated with the Fried frailty scale. ${ }^{63)}$ Although it is unknown whether the association is specific to males, the proteomics finding is consistent with the previous association using the frailty index, ${ }^{58)}$ as described above. In their proteomics study, Sathyan et al. ${ }^{62,69)}$ analyzed plasma proteomic profiles using the same technology but different de- 

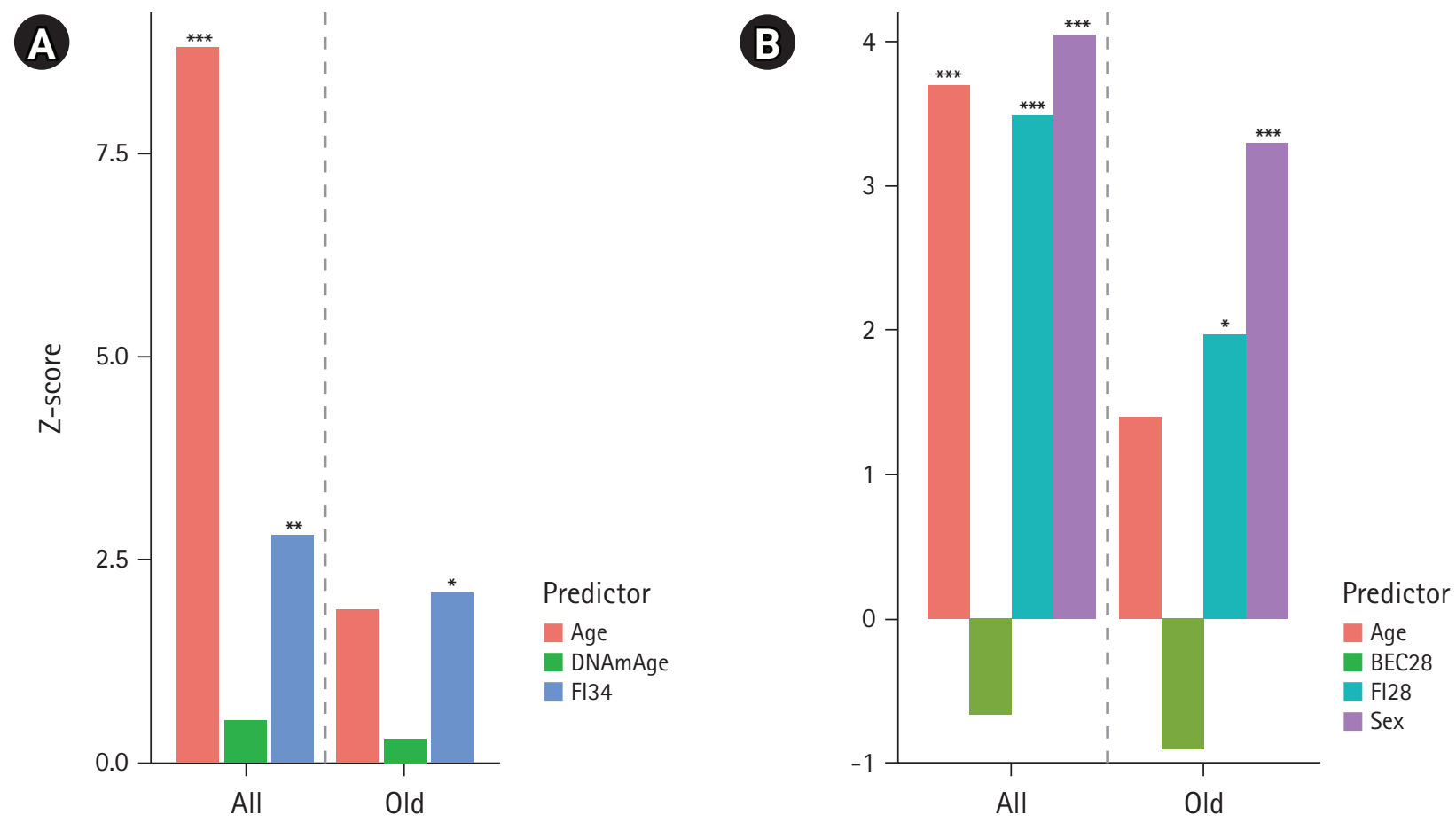

Fig. 4. Effect sizes (Z-scores) of mortality predictors in Cox proportional hazards regression analysis of 262 Louisiana Healthy Aging Study subjects. (A) Z scores of the three covariates (+sex) included in a Cox regression model for all the subjects aged 60-103 (all) and Z scores of the same covariates included in the Cox regression for the subjects aged 90-103 (old). DNAmAge is the DNA methylation age calculated according to Horvath. ${ }^{16)}$ FI34 is a frailty index based on 34 health items. ${ }^{73)}$ (B) Z scores of the four covariates included in the Cox regression for all the subjects aged 60-103 (all) and Z scores of the same covariates included in the Cox regression for the subjects aged 90-103 (old). BEC28 is the Klemera-Doubal biological age estimate using 28 health items. ${ }^{51,33)}$ FI 28 is the frailty index based on the same 28 health items. ${ }^{51,33)}{ }^{*} \mathrm{p} \leq 0.05,{ }^{* *} \mathrm{p} \leq 0.01$, ${ }^{* * *} \mathrm{p} \leq 0.001$.

pendent variables. Based on the frailty index as a dependent variable, the fatty acid-binding proteins showed the most significant association, while proteins that showed enrichment in the bioinformatic analysis were involved in lipid metabolism, cell-to-cell signaling, and interactions. ${ }^{62)}$ For chronological age, the most significantly associated proteins were pleiotrophin, WNT1-inducible-signaling pathway protein 2 , chordin-like protein 1 , transgelin, and R-spondin-1, while the bioinformatically enriched proteins were related to inflammatory response, organismal injury and abnormalities, and cell and organismal survival. ${ }^{69)}$ Thus, these two studies further demonstrated that the biological factors and pathways associated with the frailty index differed from those associated with chronological age. In other words, the biological interpretations or hypotheses to test depend on which dependent variable is used in the association analysis. Therefore, it is critical to use a reliable and accurate metric of biological age.

While single omics analysis is useful, aging is a complex phenomenon that occurs across interconnected, yet heterogeneous, biological systems. The simultaneous analysis of multiple omics data obtained from the same specimens can greatly enhance the accuracy of data analysis and interpretation. ${ }^{70,71)}$ Therefore, integrative multi-omics using a reliable measure of biological age may be a more useful approach for elucidating the biology of aging.

\section{CONCLUSION}

One fruitful approach to understanding the biology of aging is to establish a reliable measure of biological age and study its associated biological factors. Epigenetic age models, which are based on DNA methylation data, are promising in that lower epigenetic ages are associated with healthy aging and DNA methylation is considered an interface between the genome and environment. However, the biological significance and function of epigenetic model ages and model CpGs are yet to be elucidated. Frailty reflects the deteriorating physiological processes of aging. The extent of frailty is fully quantifiable by calculating the proportion of various health deficits. The frailty index is the best predictor of mortality, especially among older patients. Thus, the frailty index is a simple 
mathematical and easily rationalized method of quantifying biological age. Various biological factors are associated with frailty index, providing valuable insights into various aspects of aging. Simultaneous analysis of multi-omics datasets using the frailty index may be a fruitful approach to understanding the biology of aging.

\section{ACKNOWLEDGEMENTS}

We thank the people of Louisiana for their participation in our study. Lixin Ji participated in the research and manuscript preparation as a recipient of the Acquiring Skills and Practice in Research Excellence (ASPIRE) fellowship at Tulane School of Medicine.

\section{ACKNOWLEDGMENTS}

\section{CONFLICT OF INTEREST}

The researchers claim no conflicts of interest.

\section{FUNDING}

This work was supported by grants from the U.S. National Institutes of Health (No. AG022064, AG027905, and GM103629), the Louisiana Board of Regents through the Millennium Trust Health Excellence Fund (No. HEF[2001-2006]-02), and the Louisiana Board of Regents RC/EEP Fund through the Tulane-LSU CTRC at LSU Interim University Hospital.

\section{AUTHOR CONTRIBUTIONS}

Conceptualization, SK; Data curation, LJ, SMJ, SK; Writing-original draft, LJ, SK; Writing-review \& editing, LJ, SMJ, SK.

\section{REFERENCES}

1. Finch C. Longevity, senescence, and the genome. Chicago, IL: University of Chicago Press; 1990.

2. Benjamin H. Biologic versus chronologic age. J Gerontol 1947; 2:217-27.

3. Longo VD, Antebi A, Bartke A, Barzilai N, Brown-Borg HM, Caruso C, et al. Interventions to slow aging in humans: are we ready? Aging Cell 2015;14:497-510.

4. Colman RJ, Anderson RM, Johnson SC, Kastman EK, Kosmatka KJ, Beasley TM, et al. Caloric restriction delays disease onset and mortality in rhesus monkeys. Science 2009;325:201-4.

5. Mitchell SJ, Scheibye-Knudsen M, Longo DL, de Cabo R. Animal models of aging research: implications for human aging and age-related diseases. Annu Rev Anim Biosci 2015;3:283-303.

6. Sinclair DA, Guarente L. Extrachromosomal rDNA circles: a cause of aging in yeast. Cell 1997;91:1033-42.
7. Kimura KD, Tissenbaum HA, Liu Y, Ruvkun G. daf-2, an insulin receptor-like gene that regulates longevity and diapause in Caenorhabditis elegans. Science 1997;277:942-6.

8. Lin YJ, Seroude L, Benzer S. Extended life-span and stress resistance in the Drosophila mutant methuselah. Science 1998;282: 943-6.

9. Davidsohn N, Pezone M, Vernet A, Graveline A, Oliver D, Slomovic $\mathrm{S}$, et al. A single combination gene therapy treats multiple age-related diseases. Proc Natl Acad Sci U S A 2019;116:2350511.

10. Lopez-Otin C, Blasco MA, Partridge L, Serrano M, Kroemer G. The hallmarks of aging. Cell 2013;153:1194-217.

11. Arrojo E Drigo R, Lev-Ram V, Tyagi S, Ramachandra R, Deerinck T, Bushong E, et al. Age mosaicism across multiple scales in adult tissues. Cell Metab 2019;30:343-351. e3.

12. Tabula Muris Consortium. A single-cell transcriptomic atlas characterizes ageing tissues in the mouse. Nature 2020;583:5905.

13. Liu Z, Leung D, Thrush K, Zhao W, Ratliff S, Tanaka T, et al. Underlying features of epigenetic aging clocks in vivo and in vitro. Aging Cell 2020;19:e13229.

14. Rockwood K, Andrew M, Mitnitski A. A comparison of two approaches to measuring frailty in elderly people. J Gerontol A Biol Sci Med Sci 2007;62:738-43.

15. Hannum G, Guinney J, Zhao L, Zhang L, Hughes G, Sadda S, et al. Genome-wide methylation profiles reveal quantitative views of human aging rates. Mol Cell 2013;49:359-67.

16. Horvath S. DNA methylation age of human tissues and cell types. Genome Biol 2013;14:R115.

17. Wilson VL, Smith RA, Ma S, Cutler RG. Genomic 5-methyldeoxycytidine decreases with age. J Biol Chem 1987;262:9948-51.

18. Bollati V, Schwartz J, Wright R, Litonjua A, Tarantini L, Suh H, et al. Decline in genomic DNA methylation through aging in a cohort of elderly subjects. Mech Ageing Dev 2009;130:234-9.

19. Heyn H, Li N, Ferreira HJ, Moran S, Pisano DG, Gomez A, et al. Distinct DNA methylomes of newborns and centenarians. Proc Natl Acad Sci U S A 2012;109:10522-7.

20. Horvath S, Pirazzini C, Bacalini MG, Gentilini D, Di Blasio AM, Delledonne $\mathrm{M}$, et al. Decreased epigenetic age of PBMCs from Italian semi-supercentenarians and their offspring. Aging (Albany NY) 2015;7:1159-70.

21. Levine ME, Lu AT, Quach A, Chen BH, Assimes TL, Bandinelli $S$, et al. An epigenetic biomarker of aging for lifespan and healthspan. Aging (Albany NY) 2018;10:573-91.

22. Yang Z, Wong A, Kuh D, Paul DS, Rakyan VK, Leslie RD, et al. Correlation of an epigenetic mitotic clock with cancer risk. Genome Biol 2016;17:205. 
23. Lu AT, Quach A, Wilson JG, Reiner AP, Aviv A, Raj K, et al. DNA methylation GrimAge strongly predicts lifespan and healthspan. Aging (Albany NY) 2019;11:303-27.

24. Marioni RE, Shah S, McRae AF, Chen BH, Colicino E, Harris $\mathrm{SE}$, et al. DNA methylation age of blood predicts all-cause mortality in later life. Genome Biol 2015;16:25.

25. Lin Q, Weidner CI, Costa IG, Marioni RE, Ferreira MR, Deary IJ, et al. DNA methylation levels at individual age-associated CpG sites can be indicative for life expectancy. Aging (Albany NY) 2016;8:394-401.

26. Zhang Y, Wilson R, Heiss J, Breitling LP, Saum KU, Schottker B, et al. DNA methylation signatures in peripheral blood strongly predict all-cause mortality. Nat Commun 2017;8:14617.

27. Perna L, Zhang Y, Mons U, Holleczek B, Saum KU, Brenner H. Epigenetic age acceleration predicts cancer, cardiovascular, and all-cause mortality in a German case cohort. Clin Epigenetics 2016;8:64.

28. Quach A, Levine ME, Tanaka T, Lu AT, Chen BH, Ferrucci L, et al. Epigenetic clock analysis of diet, exercise, education, and lifestyle factors. Aging (Albany NY) 2017;9:419-46.

29. Lovkvist C, Dodd IB, Sneppen K, Haerter JO. DNA methylation in human epigenomes depends on local topology of CpG sites. Nucleic Acids Res 2016;44:5123-32.

30. Moore AZ, Hernandez DG, Tanaka T, Pilling LC, Nalls MA, Bandinelli S, et al. Change in epigenome-wide DNA methylation over 9 years and subsequent mortality: results from the InCHIANTI study. J Gerontol A Biol Sci Med Sci 2016;71:102935.

31. Greenberg MV, Bourc'his D. The diverse roles of DNA methylation in mammalian development and disease. Nat Rev Mol Cell Biol 2019;20:590-607.

32. Day K, Waite LL, Alonso A, Irvin MR, Zhi D, Thibeault KS, et al. Heritable DNA methylation in CD4+ cells among complex families displays genetic and non-genetic effects. PLoS One 2016; Oct 28;11(10):e0165488.

33. Kim M, Costello J. DNA methylation: an epigenetic mark of cellular memory. Exp Mol Med 2017;49:e322.

34. Feldman N, Gerson A, Fang J, Li E, Zhang Y, Shinkai Y, et al. G9a-mediated irreversible epigenetic inactivation of Oct-3/4 during early embryogenesis. Nat Cell Biol 2006;8:188-94.

35. Bahar Halpern K, Vana T, Walker MD. Paradoxical role of DNA methylation in activation of FoxA2 gene expression during endoderm development. J Biol Chem 2014;289:23882-92.

36. Dor Y, Cedar H. Principles of DNA methylation and their implications for biology and medicine. Lancet 2018;392:777-86.

37. Shokhirev MN, Johnson AA. Modeling the human aging transcriptome across tissues, health status, and sex. Aging Cell 2021;20:e13280.

38. Woodhouse KW, Wynne H, Baillie S, James OF, Rawlins MD. Who are the frail elderly? QJ Med 1988;68:505-6.

39. Gill TM, Gahbauer EA, Han L, Allore HG. Trajectories of disability in the last year of life. N Engl J Med 2010;362:1173-80.

40. Buchner DM, Wagner EH. Preventing frail health. Clin Geriatr Med 1992;8:1-17.

41. Fried LP, Tangen CM, Walston J, Newman AB, Hirsch C, Gottdiener J, et al. Frailty in older adults: evidence for a phenotype. J Gerontol A Biol Sci Med Sci 2001;56:M146-56.

42. Rolfson DB, Majumdar SR, Tsuyuki RT, Tahir A, Rockwood K. Validity and reliability of the Edmonton Frail Scale. Age Ageing 2006;35:526-9.

43. Rockwood K, Stadnyk K, MacKnight C, McDowell I, Hebert R, Hogan DB. A brief clinical instrument to classify frailty in elderly people. Lancet 1999;353:205-6.

44. Rockwood $\mathrm{K}$, Theou $\mathrm{O}$. Using the clinical frailty scale in allocating scarce health care resources. Can Geriatr J 2020;23:210-5.

45. Young RL, Smithard DG. The clinical frailty scale: do staff agree? Geriatrics (Basel) 2020;5:40.

46. Hewitt J, Carter B, Vilches-Moraga A, Quinn TJ, Braude P, Verduri $A$, et al. The effect of frailty on survival in patients with COVID-19 (COPE): a multicentre, European, observational cohort study. Lancet Public Health 2020;5:e444-e451.

47. Reichart D, Rosato S, Nammas W, Onorati F, Dalen M, Castro L, et al. Clinical frailty scale and outcome after coronary artery bypass grafting. Eur J Cardiothorac Surg 2018;54:1102-9.

48. Searle SD, Mitnitski A, Gahbauer EA, Gill TM, Rockwood K. A standard procedure for creating a frailty index. BMC Geriatr $2008 ; 8: 24$

49. Mitnitski AB, Mogilner AJ, Rockwood K. Accumulation of deficits as a proxy measure of aging. ScientificWorldJournal 2001; 1:323-36.

50. Kulminski AM, Ukraintseva SV, Culminskaya IV, Arbeev KG, Land KC, Akushevich L, et al. Cumulative deficits and physiological indices as predictors of mortality and long life.J Gerontol A Biol Sci Med Sci 2008;63:1053-9.

51. Kim S, Fuselier J, Welsh DA, Cherry KE, Myers L, Jazwinski SM. Feature selection algorithms enhance the accuracy of frailty indexes as measures of biological age. J Gerontol A Biol Sci Med Sci 2021;76:1347-55.

52. Kim S, Jazwinski SM. Quantitative measures of healthy aging and biological age. Healthy Aging Res 2015;4:26.

53. Klemera P, Doubal S. A new approach to the concept and computation of biological age. Mech Ageing Dev 2006;127:240-8.

54. Levine ME. Modeling the rate of senescence: can estimated biological age predict mortality more accurately than chronological 
age? J Gerontol A Biol Sci Med Sci 2013;68:667-74.

55. Wilson MM, Morley JE. Invited review: aging and energy balance. J Appl Physiol (1985) 2003;95:1728-36.

56. Kim S, Welsh DA, Ravussin E, Welsch MA, Cherry KE, Myers L, et al. An elevation of resting metabolic rate with declining health in nonagenarians may be associated with decreased muscle mass and function in women and men, respectively. J Gerontol A Biol Sci Med Sci 2014;69:650-6.

57. Kim S, Myers L, Ravussin E, Cherry KE, Jazwinski SM. Single nucleotide polymorphisms linked to mitochondrial uncoupling protein genes UCP2 and UCP3 affect mitochondrial metabolism and healthy aging in female nonagenarians. Biogerontology 2016;17:725-36.

58. Kim S, Simon E, Myers L, Hamm LL, Jazwinski SM. Programmed cell death genes are linked to elevated creatine kinase levels in unhealthy male nonagenarians. Gerontology 2016;62: 519-29.

59. Baird MF, Graham SM, Baker JS, Bickerstaff GF. Creatine-kinase- and exercise-related muscle damage implications for muscle performance and recovery. J Nutr Metab 2012;2012:960363.

60. Ruby JG, Wright KM, Rand KA, Kermany A, Noto K, Curtis D, et al. Estimates of the heritability of human longevity are substantially inflated due to assortative mating. Genetics 2018; 210:1109-24.

61. Shamsi KS, Pierce A, Ashton AS, Halade DG, Richardson A, Espinoza SE. Proteomic screening of glycoproteins in human plasma for frailty biomarkers. J Gerontol A Biol Sci Med Sci 2012; 67:853-64.

62. Sathyan S, Ayers E, Gao T, Milman S, Barzilai N, Verghese J. Plasma proteomic profile of frailty. Aging Cell 2020;19:e13193.

63. Landino K, Tanaka T, Fantoni G, Candia J, Bandinelli S, Ferrucci L. Characterization of the plasma proteomic profile of frailty phenotype. Geroscience 2021;43:1029-37.
64. Gomez-Cabrero D, Walter S, Abugessaisa I, Minambres-Herraiz $\mathrm{R}$, Palomares LB, Butcher L, et al. A robust machine learning framework to identify signatures for frailty: a nested case-control study in four aging European cohorts. Geroscience 2021;43: 1317-29.

65. Darvin K, Randolph A, Ovalles S, Halade D, Breeding L, Richardson A, et al. Plasma protein biomarkers of the geriatric syndrome of frailty.J Gerontol A Biol Sci Med Sci 2014;69:182-6.

66. Zhang Y, Chatzistamou I, Kiaris H. Identification of frailty-associated genes by coordination analysis of gene expression. Aging (Albany NY) 2020;12:4222-9.

67. Prince CS, Noren Hooten N, Mode NA, Zhang Y, Ejiogu N, Becker KG, et al. Frailty in middle age is associated with frailty status and race-specific changes to the transcriptome. Aging (Albany NY) 2019;11:5518-34.

68. Kameda M, Teruya T, Yanagida M, Kondoh H. Frailty markers comprise blood metabolites involved in antioxidation, cognition, and mobility. Proc Natl Acad Sci U S A 2020;117:9483-9.

69. Sathyan S, Ayers E, Gao T, Weiss EF, Milman S, Verghese J, et al. Plasma proteomic profile of age, health span, and all-cause mortality in older adults. Aging Cell 2020;19:e13250.

70. Cavill R, Jennen D, Kleinjans J, Briede JJ. Transcriptomic and metabolomics data integration. Brief Bioinform 2016;17:891901.

71. Mirza B, Wang W, Wang J, Choi H, Chung NC, Ping P. Machine learning and integrative analysis of biomedical big data. Genes (Basel) 2019;10:87.

72. Kim S, Myers L, WyckoffJ, Cherry KE, Jazwinski SM. The frailty index outperforms DNA methylation age and its derivatives as an indicator of biological age. Geroscience 2017;39:83-92.

73. Kim S, Welsh DA, Cherry KE, Myers L, Jazwinski SM. Association of healthy aging with parental longevity. Age (Dordr) 2013; 35:1975-82. 